Programa de trasplante de células hematopoyéticos. Departamento de Hematología y Oncología. Escuela de Medicina. Facultad de Medicina, Pontificia Universidad Católica de Chile. Santiago, Chile. aEnfermera.

Los autores declaran que no existe conflicto de intereses alguno.

Recibido el 29 de marzo de 2016 aceptado el 9 de agosto de 2016 .

Correspondencia a: Dr. Mauricio Sarmiento M. Departamento de Hematología y Oncología. Pontificia Universidad Católica de Chile. Lira $854^{\circ}$ piso. Santiago, Chile. mauriciosarmiento@hotmail.com

\section{Ajuste de intensidad para el trasplante hematopoyético alogénico en leucemia aguda}

\author{
MAURICIO SARMIENTO M., PABLO BERTÍN C., VERÓNICA JARA ${ }^{\mathrm{a}}$, \\ MAURICIO OCQUETEAU T., PABLO RAMÍREZ V.
}

\section{Intensity adjustment of hematopoietic alogeneic transplantation in acute leukemia}

Background: The intensity of conditioning chemotherapy and radiotherapy in hematopoietic stem cell transplantation (HSCT) varies according to several factors including the patient's age, pre-existing conditions and performance status. Myeloablative conditioning (MA) increases transplant related mortality and reduces survival in older patients. Reduced intensity conditioning (RIC) is a good option for these patients. Aim: To report our experience with HSCT in patients of different ages with acute leukemia. Material and Methods: Retrospective analysis of 115 allogeneic HSCT performed in patients with acute myeloid or lymphoblastic leukemia. Results: We analyzed the cohort of patients in groups according to age at transplantation: younger than 40 years $(n=74), 41$ to 50 years $(n=25)$ and older than 51 years of age $(n=16)$. Overall survival $(O S)$, Disease free survival (DFS) and relapse at five years were similar in both groups of patients younger than 50 years (OS 40 and 44\% respectively, DFS 38 and $42 \%$ respectively and relapse $40 \%$ and $34 \%$ respectively, $p=N S$ ). Patients over 51 years had a five years OS of $12 \%$. However when we analyzed those patients by date and conditioning we found that patients who were treated with MA regimens in the first decade of the transplant program (before 2000) had lower OS compared to those treated after 2000 with RIC (five years OS 49\% and 12\% respectively, $p<0.01)$. No significant differences in terms of OS, recurrence or incidence of graft-versus-host disease were found when comparing groups under 40 years, between 41 and 50 years and older than 51 years treated only with RIC. Conclusions: RIC provides the possibility of HSCT in older patients with rates comparable to those obtained in younger patients successfully treated with MA conditioning.

(Rev Med Chile 2016; 144: 1112-1118)

Key words: Aged; Hematopoietic Stem Cell Transplantation; Leukemia Myeloid, Acute; Precursor Cell Lymphoblastic Leukemia-Lymphoma.
$\mathrm{E}$ 1 trasplante hematopoyético alogénico (AloTH) ofrece la posibilidad de curación al reemplazar la hematopoyesis defectuosa e introducir efecto injerto contra leucemia ${ }^{1,2}$. El esquema de acondicionamiento para el trasplante mieloablativo (AMA) tiene alta eficiencia en eliminar células tumorales a expensas de toxicidad, sobre todo en pacientes mayores. El acondiciona- miento de intensidad reducida (AIR) ha permitido realizar AloTH en pacientes mayores con tasas de éxito comparables a las obtenidas en pacientes menores trasplantados con $\mathrm{AMA}^{3}$.

En nuestro país, el trasplante ha aumentado progresivamente y las experiencias en mieloma múltiple ${ }^{4}$, enfermedad de Hodgkin ${ }^{5}$, leucemia linfoblástica aguda ${ }^{7}$ cordón umbilical como fuente 
alternativa de donantes ${ }^{6}$ han sido comparables con los centros de referencia. Sin embargo, no tenemos información con respecto al AloTH en leucemias agudas de acuerdo a la edad. Nuestro objetivo fue analizar el impacto de la edad en pacientes adultos trasplantados por leucemias agudas de acuerdo a la intensidad del acondicionamiento.

\section{Pacientes y Métodos}

\section{Muestra}

Se realizó un análisis de la base de datos del programa de AloTH de nuestra facultad. Desde 1993 hasta 2015 se realizaron 363 trasplantes en adultos, siendo1 15 AloTH en leucemias agudas. Se recopilaron datos demográficos, tipo de donante, acondicionamiento, celularidad infundida, prendimiento de granulocitos y plaquetas, infecciones, enfermedad de injerto contra huésped (EICH), causa de muerte y tiempo de sobrevida libre de enfermedad (SLE) y sobrevida global (SG).

\section{Protocolo pre-trasplante}

Los pacientes candidatos a AloTH fueron evaluados de forma protocolizada con: a) espirometría y difusión pulmonar (DLCO), ecocardiografía, análisis suprarrenal, tiroideo, paratiroideo y gestacional; b) selección del donante con genotipificación HLA A, B, C, DR en resolución intermedia o alta, según el tipo de trasplante; c) enfermedades infecciosas con serología para herpes viridae (virus Epstein Barr, citomegalovirus, herpes simplex 1 y 2 y varicela zoster), virus de hepatitis A, B y C, VIH, sarampión, rubéola, virus de parotiditis, toxoplasma y estudio parasitario de heces y d) estado de la leucemia previo al trasplante con estudio medular clasificando en remisión completa con ausencia de células inmaduras o blastos por citología y citometría de flujo; remisión parcial, cuando hubo presencia de células inmaduras, pero en menor magnitud que al diagnóstico y enfermedad refractaria o recaída, cuando se encontró ausencia de respuesta según criterios previamente establecidos ${ }^{8}$. En nuestro programa no se realizan trasplantes en pacientes con enfermedad activa en leucemia aguda.

\section{Movilización de precursores hematopoyéticos}

Una vez que el paciente estuvo en el penúltimo día de la preparación del trasplante, se realizó la recolección de precursores hematopoyéticos del donante, previa estimulación con filgrastim en dosis de $600 \mathrm{mcg}$ sc/día por 5 días, seguido de proceso de leucoaferesis por vía periférica para obtener dosis mínima de 2x10 $0^{6}$ CD34/kg. El 95\% de los pacientes de nuestro programa son trasplantados con productos de recolección de aféresis de sangre periférica del donante. El 5\% restante, con médula ósea extraída por punción en pabellón.

\section{Quimioterapia de acondicionamiento}

Antes del año 2000, los pacientes recibieron AMA. Los esquemas usados fueron:

a) CY-TBI: ciclofosfamida endovenosa (EV) 60 $\mathrm{mg} / \mathrm{kg} /$ día por dos días e irradiación corporal total 1.320 cgy durante 4 días.

b) BU-CY: Busulfan oral $1 \mathrm{mg} / \mathrm{kg}$ cada $6 \mathrm{~h}$ por 4 días y ciclofosfamida EV $60 \mathrm{mg} / \mathrm{kg} /$ día por dos días.

c) BU-MEL-Tiotepa: Busulfan oral $1 \mathrm{mg} / \mathrm{kg}$ cada $6 \mathrm{~h}$ por 4 días, melfalan EV $140 \mathrm{mg} / \mathrm{m}^{2}$ dosis única y tiotepa $\mathrm{EV} 5 \mathrm{mg} / \mathrm{kg} /$ día por 2 días.

Después del año 2000, los pacientes mayores de 50 años recibieron AIR, tanto para donantes compatibles como haploidénticos:

a) CY-FLU-TBI: Ciclofosfamida EV $50 \mathrm{mg} / \mathrm{kg}$ dosis única, fludarabina EV $40 \mathrm{mg} / \mathrm{m}^{2}$ cada día por 5 días e irradiación corporal total 200 cgy dosis única.

b) Haplotrasplante con AIR: Ciclofosfamida EV $14,5 \mathrm{mg} / \mathrm{kg} /$ día por dos días, fludarabina EV $40 \mathrm{mg} / \mathrm{m}^{2}$ día por 5 días e irradiación corporal total 200 cgy dosis única. La ciclofosfamida se administró nuevamente el día $+3 \mathrm{y}+4$ a dosis de $50 \mathrm{mg} / \mathrm{kg} /$ día.

Todos los pacientes que recibieron ciclofosfamida tuvieron hidratación EV y mesna a dosis equivalentes.

\section{Medidas de soporte}

Durante el período de aplasia, los pacientes recibieron transfusiones para mantener plaquetas $>20.000 /$ uL y hemoglobina $>8 \mathrm{~g} \%$ y recibieron filgrastim $300 \mathrm{mcg} /$ día hasta lograr recuentos de neutrofilos $>500 / \mathrm{uL}$. El tratamiento antimicrobiano preventivo consistió en aciclovir 250 mg EV o $800 \mathrm{mg}$ vía oral cada $12 \mathrm{~h}$ y fluconazol $400 \mathrm{mg}$ día. Ante neutropenia febril, se realizó estudio y tratamiento según guías institucionales. Brevemente, ante temperatura mayor a $38^{\circ} \mathrm{C}$, se 
realizaron hemocultivo y urocultivo y radiografía de tórax; luego, se inició tratamiento antibiótico con cefalosporinas de $3^{\text {ra }}$ generación, aminoglucósido y vancomicina. Ante persistencia de fiebre a $72 \mathrm{~h}$ de iniciado el esquema, se solicitó tomografía computada (TAC) de cavidades paranasales, tórax, abdomen y pelvis y se realizaron tests de infección fúngica. El ajuste antimicrobiano fue decidido según los hallazgos radiológicos o de los cultivos. En caso de reactivación de citomegalovirus, se inició terapia preventiva con ganciclovir o foscarnet.

Todos los pacientes recibieron tratamiento analgésico opiáceo en caso de mucositis grado 2 a 4 graves y nutrición parenteral según necesidad.

\section{Profilaxis de enfermedad de injerto contra huésped}

El esquema de prevención de EICH aguda en trasplante de donante hermano compatible y no relacionado compatible consistió en ciclosporina ajustada para niveles plasmáticos entre 250 y 400 ng/uL y metrotexate $15 \mathrm{mg} / \mathrm{m}^{2} \mathrm{EV}$ día 1 y $10 \mathrm{mg} /$ $\mathrm{m}^{2}$ días $+3,+6,+11$. En trasplante haploidéntico consistió en ciclofosfamida post trasplante los días +3 y +4 , y desde el día +5 micofenolato mofetil oral $10-15 \mathrm{mg} / \mathrm{kg}$ cada $12 \mathrm{~h}$ y tacrolimus $0,03 \mathrm{mg} /$ $\mathrm{kg} /$ dia EV en infusión continua, con cambio a terapia oral según tolerancia para lograr niveles plasmáticos entre 5 y $15 \mathrm{ng} / \mathrm{uL}$.

\section{Definiciones de complicaciones post trasplante}

La EICH se definió según el tiempo de aparición y desarrollo de las características clínicas. Así, los pacientes con EICH en los primeros 100 días post trasplante se clasificaron como EICH agudos y aquellos con manifestaciones luego de ese período fueron clasificados como EICH crónico. La sobrevida global se definió como el tiempo transcurrido entre el día del trasplante y la fecha de muerte o la fecha del último control. La SLE se definió como el tiempo transcurrido entre el día del trasplante y la fecha de recaída. La recaída se definió como la evidencia de reaparición de blastos. La mortalidad no relacionada a recaída (NRM) se definió como cualquier causa de muerte no asociada a recidiva de la enfermedad, incluyendo las asociadas a complicaciones directamente relacionadas con el trasplante.

\section{Análisis estadístico}

La prueba de Shapiro-Wilk evaluó la normalidad de las variables cuantitativas. Las caracterís- ticas demográficas y basales fueron presentadas utilizando media, porcentaje y rangos. Las comparaciones entre las variables se realizaron con el método de Chi cuadrado. Las probabilidades de SG se calcularon con el método de Kaplan-Meier y las comparaciones con la prueba de log-rank. Los análisis de recaída, NRM y EICH se realizaron en un marco de riesgos competitivos mediante el estimador no paramétrico de incidencia acumulada. Para estimar el efecto de la edad en la SG, se ajustó el modelo de Kaplan-Meier de acuerdo a los rangos de edad comprendidos en menores de 40 años, entre 41 y 50 años y mayores de 51 años. El efecto de los acontecimientos que tuvieron lugar durante el seguimiento y después del trasplante, como la EICH aguda, fueron analizados como una covariable dependiente del tiempo. El software usado fue SPSS.V.15 (IBM Software, USA) y Prisma Software V 6.0.1 (GraphPad software, USA). Las diferencias se consideraron significativas para valores de $\mathrm{p}<0,05$ con intervalos de confianza (IC) del 95\%.

\section{Resultados}

\section{Características de los pacientes}

Como se describe en la Tabla 1, 43\% de los pacientes fueron mujeres, con una edad promedio de 35 años para toda la cohorte (rango 17-60). El $54 \%$ tenía leucemia mieloide aguda (LMA) y el $46 \%$ leucemia linfoblástica aguda (LLA), en su mayoría en primera remisión completa. El 89\% de los pacientes tuvo donante compatible HLA idéntico.

\section{Acondicionamiento}

Durante la primera década del programa (1993-2000) se realizaron 17 trasplantes, todos AMA, $70 \%$ con esquema Cy-TBI, 25\% con esquema BU-CY y $5 \%$ con esquema BU-MEL-Tiotepa. Después del año 2000 se realizaron 98 trasplantes, de los cuales fueron 12 (12\%) BU-CY, $5(5 \%)$ BU-CY-TBI, 48 (50\%) CY-TBI, 17 (17\%) AIR CYFLU-TBI y 16 (16\%) con esquema haploidéntico, de los cuales 13 fueron mieloablativos y 6 AIR.

\section{Movilización y recolección de precursores hematopoyéticos}

Como se describe en la Tabla 2, la recolección de precursores se logró en un primer intento en 93\% de los casos. Siete por ciento requirió dos aféresis 
Ajuste de intensidad para el trasplante en leucemias agudas - M. Sarmiento et al

Tabla 1. Características basales de los pacientes

\begin{tabular}{|c|c|c|}
\hline Número de pacientes & $\mathrm{n}=115$ & \\
\hline Edad al trasplante & 35 años (rango 17-60) & \\
\hline Género & $\begin{array}{l}\text { Masculino } \\
\text { Femenino }\end{array}$ & $\begin{array}{l}65(75 \%) \\
50(25 \%)\end{array}$ \\
\hline \multirow[t]{5}{*}{ Diagnóstico } & $\begin{array}{l}\text { Leucemia mieloide aguda } \\
\qquad \begin{array}{l}\text { M1 } \\
\text { M2 } \\
\text { M3 refractaria } \\
\text { M4 } \\
\text { M5 } \\
\text { M6 } \\
\text { Secundaria a mielodisplasia } \\
\text { M0 y/o indiferenciada }\end{array}\end{array}$ & $\begin{aligned} 66 & (58 \%) \\
6 & (10 \%) \\
10 & (15 \%) \\
1 & (1,5 \%) \\
11 & (16,5 \%) \\
4 & (6 \%) \\
2 & (3 \%) \\
12 & (18 \%) \\
19 & (28,5 \%)\end{aligned}$ \\
\hline & Bifenotipíca & $1 \quad(1,5 \%)$ \\
\hline & Leucemia linfoblástica aguda & $49(42 \%)$ \\
\hline & Philadelphia $(+)$ & $26(52 \%)$ \\
\hline & Philadelphia (-) & $23(48 \%)$ \\
\hline Status de la enfermedad al trasplante & $\begin{array}{l}1^{\text {ra }} \text { remisión completa } \\
2^{\text {da }} \text { remisión completa } \\
3^{\text {ra }} \text { remisión completa }\end{array}$ & $\begin{array}{lr}60 & (55 \%) \\
45 & (40 \%) \\
10 & (5 \%)\end{array}$ \\
\hline Quimioterapia de condicionamiento & $\begin{array}{l}\text { Bu-Mel-Tiotepa }{ }^{1} \\
\text { Cy-Flu-TBI } \\
\text { Cy-TBI } \\
\text { Bu-Cy } \\
\text { Bu-Cy-TBI }{ }^{5}\end{array}$ & $\begin{array}{rr}2 & (1 \%) \\
25 & (22 \%) \\
68 & (60 \%) \\
15 & (12 \%) \\
5 & (5 \%)\end{array}$ \\
\hline Tipo de condicionamiento & $\begin{array}{l}\text { Mieloablativo } \\
\text { Intensidad reducida }\end{array}$ & $\begin{array}{ll}98 & (80 \%) \\
17 & (20 \%)\end{array}$ \\
\hline Profilaxis de EICH & $\begin{array}{l}\text { Ciclosporina-metrotexato } \\
\text { Tacrolimus-micofenolato/PTCY }\end{array}$ & $\begin{array}{ll}97 & (84 \%) \\
18 & (16 \%)\end{array}$ \\
\hline Tipo de donante & $\begin{array}{l}\text { HLA idéntico relacionado } \\
\text { HLA idéntico no relacionado } \\
\text { Haploidéntico }\end{array}$ & $\begin{array}{ll}89 & (77 \%) \\
11 & (10 \%) \\
15 & (13 \%)\end{array}$ \\
\hline Fuente de progenitores & $\begin{array}{l}\text { Médula ósea } \\
\text { Sangre periférica }\end{array}$ & $\begin{array}{r}6 \\
109 \%) \\
109(95 \%)\end{array}$ \\
\hline
\end{tabular}

'Busulfan-melfalan-tiotepa, ${ }^{2}$ Ciclofosfamida-fludarabina-irradiación corporal total, ${ }^{3}$ Ciclofosfamida-irradiación corporal total, ${ }^{4}$ Busulfan-cyclophosphamide, ${ }^{5}$ Busulfan-ciclofosfamida-irradiación corporal total, ${ }^{6}$ ciclofosfamida post trasplante.

Tabla 2. Características del trasplante y resultados

\begin{tabular}{|lll|}
\hline & Media & Rango \\
\hline CD34 infundidos $\times 10^{6} / \mathrm{kg}$ de peso & 4,9 & $1-11$ \\
\hline Día de prendimiento granulocítico & 15 & $8-26$ \\
\hline Día de prendimiento plaquetario & 21 & $8-193$ \\
\hline Incidencia acumulada de EICH aguda a 100 días & $21 \% \mathrm{Cl} 95 \% 18-36$ & \\
\hline Incidencia acumulada de EICH crónico a 1 año & $17 \% \mathrm{Cl} 95 \% 14-35$ & $42 \%$ \\
\hline Recaída & & \\
\hline
\end{tabular}


y $1 \%$ requirió 3 aféresis para lograr la celularidad deseada. En promedio los pacientes recibieron 4,9 x $10^{6} \mathrm{CD} 34 / \mathrm{kg}$ de peso (rango $1-11 \times 10^{6}$ ).

\section{Complicaciones post trasplante y prendimiento}

Todos los pacientes tuvieron neutropenia febril en el período de aplasia, con infección bacteriana documentada en $60 \%$ de los pacientes. La respuesta al antibiótico fue satisfactoria resolviendo la infección en $88 \%$ de los pacientes, con $12 \%$ de infecciones graves que ameritaron tratamiento en cuidados intensivos. En $7 \%$ de los pacientes se documentó infección fúngica invasora. Todos los pacientes recibieron tratamiento opiáceo por mucositis grado $>2$, con necesidad de uso de nutrición parenteral. El prendimiento del injerto granulocítico fue, en promedio, a los 15 días (rango 8-26) y del plaquetario, 21 días (rango 8-193).

\section{Incidencia de EICH aguda y crónica}

La incidencia acumulada de EICH aguda grado 2-4 a 100 días fue de $21 \%(n=19)$ con un tiempo promedio de aparición de los primeros síntomas a los 24 días (rango 5-60). La incidencia de EICH crónica fue de $17 \%(\mathrm{n}=17)$ con un tiempo de aparición de los primeros síntomas a 240 días (rango 100-480). En cuanto al compromiso del EICH crónico, en $70 \%$ fue localizado y en $30 \%$ fue extenso. No hubo diferencias entre la incidencia de EICH al ajustar por edad ni por intensidad del acondicionamiento.

\section{Sobrevida global}

La SG de los pacientes trasplantados por leucemia aguda a 5 años fue de $45 \%, 60 \%$ y $12 \%$ en los menores de 40 años, entre 41 y 50 años y mayores 51 años, respectivamente $(\mathrm{p}=0,0036$, Figura 1). Al analizar los enfermos mayores de 51 años según la intensidad de acondicionamiento, se encontró que aquellos tratados con AIR tuvieron una SG de 55\%, mientras que los tratados con AMA tuvieron una sobrevida estimada de $10 \%$ a 5 años $(\mathrm{p}=0,008)$. Al comparar los pacientes menores de 40 años con los pacientes entre 41 y 50 años tratados con AMA con aquellos mayores de 51 años tratados con AIR, no se encontraron diferencias significativas ( $\mathrm{p}=0,38$, Figura 2). Para establecer si la SG estadísticamente similar en los pacientes mayores de 50 años y menores era por efecto netamente de la edad o si era influido por el acondicionamiento, se realizó una comparación de SG en los pacientes tratados con AIR en los diferentes grupos sin encontrar diferencias significativas $(\mathrm{p}=0,45)$.

\section{Mortalidad por trasplante y recaídas}

Cincuenta y nueve pacientes fallecieron. La muerte por recaída se encontró en $42 \%(\mathrm{n}=25)$, y la muerte no relacionada a recaída en $48 \%$ $(\mathrm{n}=34)$. Dentro de estos últimos, las causas fueron EICH aguda o crónica $(\mathrm{n}=4)$, sangrado en sistema nervioso central $(n=1)$, sepsis $(n=26)$ y toxicidad del acondicionamiento $(n=3)$. No hubo diferencias en cuanto a la causa de muerte en los diferentes grupos de edad. Así, en el grupo de menores de 40 años $(n=73)$ hubo 14 muertes por sepsis, 4 por EICH, 17 por recaída, 2 por toxicidad del acondicionamiento y 1 por sangrado en el sistema nervioso central; en el grupo de pacientes con edades entre 41 y 50 años $(n=25)$ hubo

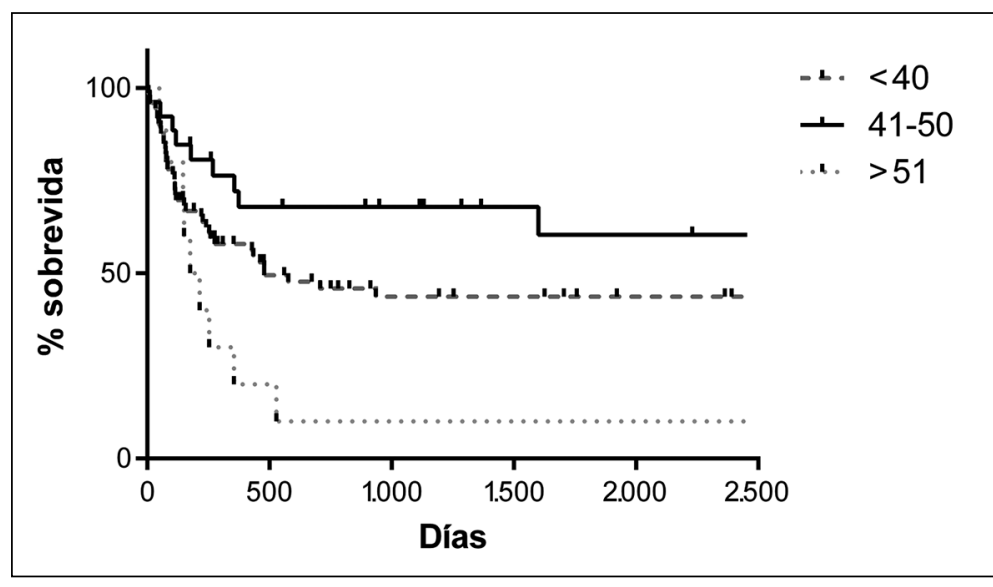

Figura 1. Sobrevida global en pacientes con leucemia aguda tratados con trasplante alogénico de acuerdo a la edad al momento del trasplante. 


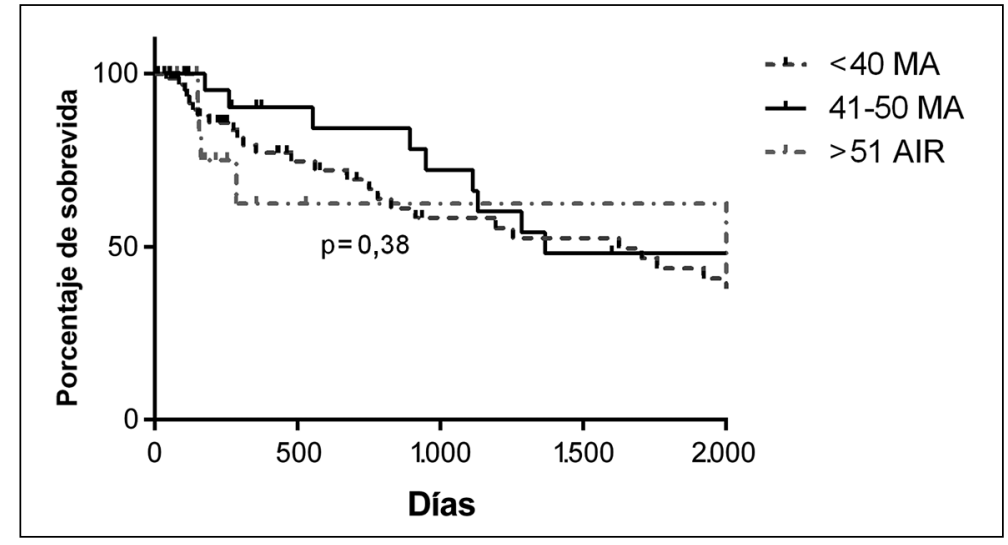

Figura 2. Sobrevida global en pacientes con leucemia aguda tratados con trasplante alogénico de acuerdo a la edad e intensidad del acondicionamiento.
6 muertes por sepsis y 4 por recaída y en el grupo de pacientes mayores de 51 años $(n=16)$ hubo 6 muertes por sepsis, 4 por recaída $(\mathrm{p}=0,516)$.

\section{Discusión}

La edad de los pacientes que reciben trasplante ha cambiado durante los últimos años. Cuando el uso de AMA fue la única opción, la edad al trasplante era entre 25 y 30 años ; sin embargo, la mayoría de los pacientes al debut de su hemopatía tienen edad sobre 50 años. Así, si se restringe el trasplante a pacientes menores de 50 años, sólo $8 \%$ de los pacientes se beneficiarían, mientras que si se aplica el trasplante a mayores de 50 años el porcentaje de tratados sería $38 \%{ }^{10}$. Si bien el tratamiento con AMA es tóxico con mala tolerancia en edades avanzadas, el tratamiento con AIR tiene menores tasas de complicaciones y es aplicable a pacientes cada vez mayores ${ }^{11}$.

En la experiencia de nuestro programa de trasplantes se han obtenido resultados que reproducen la información de los grandes centros de AloTH. En nuestro estudio encontramos que los pacientes mayores tratados con AMA tuvieron una alta morbilidad con sobrevida menor a $15 \%$, lo cual es inferior a lo encontrado en los pacientes menores de 50 años tratados con quimioterapia de igual intensidad. Sin embargo, con la implementación de AIR en pacientes mayores, la sobrevida mejoró e incluso superó a la de pacientes más jóvenes tratados con AMA, manteniendo tasas de EICH y recaídas aceptables. Esta misma experiencia ha sido reportada antes. El grupo de Minnesota reportó los resultados de trasplante en pacientes mayores de 70 años, encontrando que con el AIR se obtuvo una sobrevida global de $60 \%$ a 2 años con una incidencia de EICH crónico de $10 \%$ y sobrevida en pacientes entre 60 y 69 años igual que en los mayores de $70^{13}$. Blaise et $\mathrm{al}^{14}$ mostraron que el trasplante haploidéntico puede ser realizado en mayores de 50 años, con baja incidencia de EICH y sobrevida global a 2 años de $70 \%$. El grupo de Baltimore, pionero en el desarrollo del trasplante haploidéntico, mostró que en 119 pacientes tratados con AIR, con edades entre los 50 y 59 años, 60 a 69 años y 70 a 75 tuvieron la misma SLE y $\mathrm{SG}^{15}$. Sin embargo, la mayoría de la información disponible es generada de estudios retrospectivos de grandes centros de registro internacional o recomendaciones de expertos de centros de referencia. Recientemente se realizó un metanálisis que mostró que no hay ninguna ventaja del AMA en cuanto a sobrevida global y que AIR es una opción efectiva en pacientes no candidatos a esquemas de alta intensidad, siempre y cuando el estado pre-trasplante sea de remisión completa ${ }^{16}$.

Las limitaciones de nuestro estudio están dadas principalmente por la naturaleza retrospectiva del análisis. Sin embargo, esto puede considerarse una fortaleza, pues dado el prolongado período de funcionamiento de nuestro programa de trasplante, muestra los resultados de este tipo de tratamiento en un mundo real fuera de los ensayos controlados.

Nuestros datos muestran que al aplicar protocolos de acondicionamiento con intensidad ajustada, selectivos e individualizados, el beneficio del trasplante puede aplicarse a un mayor número 
de enfermos. Podemos concluir, entonces, que la intensidad del acondicionamiento ajustada por la edad permite que la toxicidad del trasplante sea aceptable y que la sobrevida global sea similar en los diferentes grupos etareos. Específicamente, nuestro estudio muestra que es posible realizar AloTH en nuestro país en pacientes mayores de 50 años con el uso de AIR. Nuestros resultados son comparables con los reportados por grupos de referencia.

\section{Referencias}

1. Glodstone AH, Rowe JM. Transplantation in adult ALL. Hematology Am Soc Hematol Educ Program. 2009: 593-601.

2. Gale RP, Champlin RE. How does bone-marrow transplantation cure leukemia? Lancet 1984; 2: 28-30.

3. Champlin R, Khouri I, Shimoni A, Gajewski J, Kornblau $\mathrm{S}$, Molldrem J, et al. Harnessing graft- versus-malignancy: non-myeloablative preparative regimens for allogeneic hematopoietic transplantation, an evolving strategy for adoptive immunotherapy. Br J Haematol 2000; 111 : 18-29.

4. Sarmiento M, Lira P, Ocqueteau M, Rodríguez MA, García MJ, Jara V, et al. [Autologous hematopoietic cell transplantation in patients with multiple myeloma. Experience in 53 patients]. Rev Med Chile 2014; 142 (12): 1497-501.

5. Ramírez P, Ocqueteau M, Rodríguez A, García MJ, Sarmiento M, Ernst D, et al. Outcomes in relapsed Hodgkin's lymphoma treated with autologous and allogeneic hematopoietic cell transplantation at the Pontificia Universidad Católica de Chile. Rev Bras Hematol Hemoter 2015; 37 (3): 184-9.

6. Ramírez P, Nervi B, Bertin P, Poggi H, Lagos M, Selman C. Umbilical cord blood transplantation in hematologic diseases in patients over 15 years old: long-term experience at the Pontificia Universidad Católica de Chile. Transplant Proc 2013; 45 (10): 3734-9.

7. Ernst D, Escobar K, Bertin P, Nervi B, Jara V, Ocqueteau $\mathrm{M}$, et al. Similar outcomes in allogenic hematopoietic cell transplantation for acute lymphoblastic leukemia patients is achievable in developing Countries: single center experience at a university hospital in Chile. J Hematol Transfus 2014; 2(3): 1030-6.

8. Döhner H, Estey EH, Amadori S, Appelbaum FR, Bü- chner T, Burnett AK, et al. Diagnosis and management of acute myeloid leukemia in adults: recommendations from an international expert panel, on behalf of the European Leukemia Net Blood 2010; 115 (3): 453.

9. Kollman C, Howe CW, Anasetti C, Antin JH, Davles $\mathrm{SM}$, Filipovich AH, et al. Donor characteristics as risk factors in recipients after trasnplantation of bone marrow from unrelated donors: the effect of donor age. Blood 2001; 98: 2043-51.

10. Aul C, Gattermann N, Scheider W. Age-related incidence and other epidemiological aspects of myelodisplastic syndromes. Br J Haematol 1992; 82: 358-67.

11. Schneidawind D, Federmann B, Buechele C, Helwig A, Schmohl J, Vogel W, et al. Reduced-intensity conditioning with fludarabine and busulfan for allogeneic hematopoietic cell transplantation in elderly or infirm patients with advanced myeloid malignancies. Ann Hematol 2015; sep 28. epub ahead of print.

12. De la Camara R, Alonso A, Steegmann JL, Arranz R, Granados E, Rodríguez-Macías G, et al. Allogeneic hematopoietic stem cell transplantation in patients 50 years of age and older. Haematologica 2002; 87: 965-72.

13. Sandhu KS, Brunstein C, DeFor T, Bejanyan N, Arora $\mathrm{M}$, Warlick E, et al. Umbilical cord blood transplantation outcomes in acute myelogenous leucemia/myelodysplastic syndromes patients $>70$ years old. Biol Blood Marrow Transplant 2015; sept 25: s1083.

14. Blaise D, Fürst S, Crocchiolo R, El-Cheikh JM, Granata A, Harbi S, et al. Haploidentical T cell replete transplantation with post transplantation cyclophosphamide for patients in or aboce the sixth decade of age compared with allogeneic hematopoietic stem cell transplantation from an human lekocyte antigen-matched related or unrelated donor. Biol Blood Marrow Transplant 2015; sept 1. s1083.

15. Kasamon YL, Bolaños-Meade IJ, Prince GT, Tsai HL, McCurdy SR, Kanakry JA, et al. Outcome of non myeloablative HLA haploidentical blood or marrow transplantation with high dose post transplantation cycophosphamide in older adults. J Clin Oncol 2015; (33): 3152-61.

16. Abdul Wahid SF, Ismail NA, Mohd-Idris MR, Jamaluddin FW, Tumian N, et al. Comparison of reduced-intensity and myeloablative conditioning regimens for allogeneic hematopoietic stem cell transplantation in patients with acute myeloid leukemia and acute lymphoblastic leukemia: a meta-analysis. Stem Cells Dev 2014; 23 (21): 2535-52. 